\title{
Metastatic malignant melanoma arising in the ovary: A case report and review of the literature
}

\author{
Amani Harris ${ }^{1}$, Norman Blumenthal ${ }^{2}$ \\ ${ }^{1}$ Blacktown and Westmead Hospitals, New South Wales, Australia \\ ${ }^{2}$ Blactown Hospital, New South Wales, Australia \\ Email: a_hares@hotmail.com, blum@bigpond.net.au
}

Received 25 May 2013; revised 28 June 2013; accepted 6 July 2013

Copyright (C) 2013 Amani Harris, Norman Blumenthal. This is an open access article distributed under the Creative Commons Attribution License, which permits unrestricted use, distribution, and reproduction in any medium, provided the original work is properly cited.

\begin{abstract}
Introduction: This article is a case report of metastatic malignant melanoma to the ovary and a review of the current literature on recommendations for diagnosis and management. Background: Metastatic involvement of the ovary from malignant melanoma is relatively uncommon and represents a diagnostic challenge. There are $\mathbf{7 4}$ cases of malignant melanoma metastasing to the ovary reported in the English literature. Malignant melanoma in the ovary is often found at autopsy, as a part of an extensive multisystemic spread of the disease and is rarely diagnosed during life. Most cases reported in the literature thus far occurred in women of reproductive age. Commonly, the history of a primary cutaneous melanoma lesion is remote, and in some cases, absent [1]. Most cases are associated with disseminated disease and carried a dismal prognosis [2].
\end{abstract}

Keywords: Melanoma; Ovary; Metastatic

\section{CASE REPORT}

A 39-year-old woman presented to the gynaecology outpatient clinic with a two week history of right sided loin pain radiating to the right iliac fossa. On examination, there was no ascites, the abdomen was soft and bimanual examination revealed bilateral adnexal masses, which were non-tender and mobile. The Pouch of Douglas was clear of any nodularity.

A pelvic ultrasound performed revealed bilateral solid and cystic adnexal masses. The right adnexal mass measured $9.8 \times 7.5 \times 7.2 \mathrm{~cm}$ and consisted of a honeycomb of multiple cystic spaces measuring up to $3.7 \mathrm{~cm}$ in diameter. There was variable appearance and thickness with a considerable solid component. The left adnexal mass was almost entirely unilocular measuring $8.1 \times 6.8$ $\times 8.0 \mathrm{~cm}$.

Abdominal ultrasound revealed a soft tissue mass adjacent to the upper pole of the right kidney measuring 46 $\times 37 \times 35 \mathrm{~mm}$ possibly representing an enlarged adrenal gland.

Computed Tomography (CT) of the abdomen and pelvis confirmed the above findings, showing a small volume of free fluid in the pelvis. No lymphadenopathy was evident in the pelvis, retroperitoneum or upper abdomen. The gallbladder, liver, spleen and pancreas were of normal appearance. Features were suggestive of ovarian cystic neoplasms, most likely mucinous cystadenoma or cystadenocarcinoma. All tumour markers were negative including: CA125, LDH, CA19.9, CEA and Beta HCG.

The patient underwent a laparotomy with right salpingoophorectomy. The right ovary was found to be reasonably mobile although there were adhesions to the Pouch of Douglas and right pelvic side wall. The ovary was easily mobilized and removed without any spill. The left ovary, in spite of the original ultrasound report, appeared normal at the time of surgery and was conserved.

The pathology reported that macroscopically, the ovarian specimen measured $85 \times 75 \times 62 \mathrm{~mm}$ with solid grey-brown interior, cystic degeneration and haemorrhage. A solid and focally villiform tumour was identified at the serosal surface and lining the wall of the ovary up to $20 \mathrm{~mm}$ in depth. A unilocular cyst was present with further two cystic structures at its surface measuring $35 \times$ $30 \times 15 \mathrm{~mm}$ and $15 \times 15 \times 15 \mathrm{~mm}$. On sectioning, the main cyst appeared to be lined by solid pale tan tumour with some papillary looking areas. The smaller cysts had a similar pattern within their lumens. Microscopically, the tumour was composed of cells which grew in sheets and formed papillae. Slides showed anaplastic epithelloid cells with Grade 3 nuclei including prominent nucleoli and a moderate amount of eosinophillic cytoplasm. The 
smaller ovarian cysts also contained the same tumour. Immunohistochemically, the tumour cells expressed S100 and MART-1 and were negative for epithelial markers. This pattern of immuno-expression confirmed a diagnosis of malignant melanoma and since no evidence of a teratoma in the ovary was ever found, the diagnosis of metastatic malignant melanoma was made.

The patient made a good recovery post procedure. After the histological diagnosis, she was clinically reassessed by a Dermatologist for a full body check which showed no evidence of cutaneous melanoma. A fine needle aspiration biopsy of the right adrenal gland also confirmed metastatic melanoma. A repeat CT scan of her chest, abdomen and pelvis was performed showing possible evidence of metastases on the left upper lobe of her lung. She was then reviewed by a medical oncologist for further treatment.

\section{DISCUSSION}

Metastatic melanoma to the ovary is a rare but reportedly lethal disease, occurring mostly in women of reproductive age. The tumour is usually unilateral and involving other organs, most commonly the lungs at time of diagnosis [1].

The ovary is a frequent site of secondary spread from extra-ovarian malignancies. Approximately $6 \%-7 \%$ of the patients presenting with suspected ovarian neoplasms are diagnosed as having metastatic disease to the ovary [3]. Ovarianmetastases, in the context of non-gynaecological malignancies commonly arise from gastrointestinal adenocarcinomas and breast cancer [3].

Malignant melanomas of the ovary are neoplasms of neuroectodermal origin that often present in diverse sites [4], seen in approximately $18 \%$ of patients with disseminated disease [5].

The mean age at diagnosis of malignant melanoma metasteses to the ovary is 38 years. It is suggested that the rich vasculature of the ovaries during reproductive years contributes to its involvement via the haematogenous route at that time [3]. The diagnosis of metastatic malignant melanoma in such an age group is devastating because of the poor prognosis despite treatment. The only figures available are by Fletcher et al. whose study is based on surgical management of metastasis to abdominal viscera, lung and brain [4]. The 5-year survival rate is reported as $10 \%$ for solitary lesions and $0 \%$ for multiple lesions [4].

The diagnosis of metastatic malignant melanoma in an ovarian mass is challenging for many reasons. Firstly, in many instances, a history of a primary melanoma lesion is remote or absent as seen in cases of a regressed cutaneous lesion or a primary undetected lesion arising in mucosal sites [1]. The average time from diagnosis of a primary lesion to metastasis in the ovary is reported as 7 years. However, documented cases of recurrence in the ovary have been reported 25 years after diagnosis of a primary lesion [4]. Secondly, metastatic melanomas assume varied appearance which poses great diagnostic difficulty upon immuno-histological examination [6]. Malignant melanoma cells are described as having numerous patterns and varied cell types. In a large study of 16 categories of tumours metastatic to the ovaries, young describes the extremely broad differential diagnosis posed by metastatic malignant melanoma in the ovary. These include steroid cell tumours, small cell tumours, sarcomas, undifferentiated carcinoma, transitional cell carcinoma and adult granulosa cell tumours [6]. As described, the histopathologic features of malignant melanoma in the ovary may resemble a wide range of primary ovarian tumours. Immunohistochemical analysis has been shown to be of great value. The S100 protein is most widely used by histopathologists. Bishop, et al. reported 94\% S100 positivity for primary and metastatic melanoma [7]. Other promising tumour markers are HMB-45 and MART-1 (anti-melanin-A) monoclonal antibody. Both have between 90\% - 100\% positivity staining for primary or metastatic melanoma, but fail to label most cases of desmoplastic malignant melanoma [5].

Most diagnoses of metastatic melanoma in the ovary are made via immunohistopathological examination postoperatively. In one case described by Moselhi, et al., the diagnosis was established preoperatively via ascitic fluid cytology [8].

The current recommendation in the limited literature available for the treatment of metastatic malignant melanoma to the ovary remains surgical. This includes adequate tumour debulking, and evaluation for local, regional and distant spread. In perimenopausal or postmenopausal women, total abdominal hysterectomy with bilateral salpingo-oophorectomy remains appropriate, unless extensive disease warrants a conservative approach. In women of reproductive age, unilateral salpingo-oophorectomy is currently the treatment of choice if the other ovary appears normal [9]. The role of adjuvant chemotherapy or radiation therapy has not been established [9]. Despite this fact, referral to a medical oncologist is crucial to discuss options of further treatment [4]. Postoperative management should always include imaging of all potential sites of metastasis, most commonly the lung [4].

\section{CONCLUSIONS}

In conclusion, this present case represents the diverse behaviour of malignant melanoma and its incidence in young women of reproductive age. It highlights the difficulty in diagnosing a malignant melanoma in the ovary 
in the absence of a previous history of melanoma. It also highlights the importance of immunohistochemical analysis for diagnostic purposes, and the role of adequate surgical debulking to achieve optimal patient prognosis. Post-operative imaging in this case revealed metastases to the adrenal gland and lung. As depicted in our case report, clinical evidence was lacking for the role of radiation or chemotherapy post operatively in improving survival rates; nevertheless, our patient was referred on to the medical oncologist for further treatment and follow up.

More researches are required to establish methods for diagnosis preoperatively and definitive post operative medical management to improve survival of a fatal disease.

\section{REFERENCES}

[1] Gupta, D., Deavers, D., Silva, E. and Malpica. A. (2004) Malignant melanoma involving the ovary: A clinicopathologic and immunohistochemichal study of 23 cases. The American Journal of Surgical Pathology, 28, 771780. doi:10.1097/01.pas.0000126786.69232.55

[2] Boustis, A., Valeri, R., Korantzis, I., et al. (2008) Delayed malignant melanoma recurrence stimulating primary ovarian cancer: Case report. World Journal of Surgical Oncology, 6, 124-129. doi:10.1186/1477-7819-6-124

[3] Moore, R., Chung, M., Granai. C., Gajewski, W. and
Steinhoff, M. (2004) Incidence of metastasis to the ovaries fronnongenital tract primary tumors. Gynecologic Oncology, 93, 87-91. doi:10.1016/j.ygyno.2003.12.039

[4] Fuller, P. (1999) Malignant melanoma of the ovary and exposure to clomiphine citrate: A case report and review of the literature. American Journal of Obstetrics \& Gynecology, 180, 1499-1503. doi:10.1016/S0002-9378(99)70045-1

[5] Curtin, J.P. and Morrow, C.P. (1992) Melanoma of the female genital tract. In: Coppelson, M., Monaghan, J.M., Morrow, C.P. and Tattersall, M.H., Eds., Gynecologic Oncology, 2nd Edition, Churchill Livingstone, London, 9, 1059-1068.

[6] Young, P. (2007) From Krukenberg to today: The ever present problems posed by metastatic tumors in the ovary, Part II. Advances in Anatomic Pathology, 14, 149-177. doi:10.1097/PAP.0b013e3180504abf

[7] Bishop, P.W., Menasce, L.P., Yates, A.J., Win, N.A. and Banerjee, S.S. (1993) An immunophenotypic survey of malignant melanomas. Histopathology, 23, 159-166. doi:10.1111/j.1365-2559.1993.tb00474.X

[8] Moselhi, M., Spencer, J., and Lane, G. (1998) Malignant melanoma metastatic to the ovary: Presentation and radiological characteristics. Gynecologic Oncology, 69, 165-168. doi:10.1006/gyno.1998.4992

[9] Banerjee, S.S. and Harris, M. (2000) Morphological and Immunophenotypic variations of malignant melanoma. Histopathology, 36, 387-402. doi:10.1046/j.1365-2559.2000.00894.X 